\title{
Baseline neutrophil-to-lymphocyte ratio predicts the prognosis of castration-resistant prostate cancer treated with abiraterone acetate
}

\author{
MASATO YASUI $^{1}$, YORIKO HASEGAWA ${ }^{2}$, TAKASHI KAWAHARA ${ }^{1}$, YOHEI KUMANO ${ }^{1}$, \\ YASUHIDE MIYOSHI ${ }^{1}$, NOBUAKI MATSUBARA ${ }^{2}$ and HIROJI UEMURA ${ }^{1}$ \\ ${ }^{1}$ Department of Urology and Renal Transplantation, Yokohama City University Medical Center, Yokohama 232-0024; \\ ${ }^{2}$ Department of Breast and Medical Oncology, National Cancer Center Hospital East, Kashiwa 277-8577, Japan
}

Received October 16, 2017; Accepted December 15, 2017

DOI: $10.3892 / \mathrm{mco} .2018 .1573$

\begin{abstract}
Abiraterone acetate (AA), a CYP17 inhibitor, now has a crucial role in the treatment of castration-resistant prostate cancer (CRPC), and previous studies have reported several prognostic clinical factors for AA treatment. The neutrophil-to-lymphocyte ratio (NLR) has also been investigated for a CRPC treatments in a few reports, however it has not been identified to be a prognostic factor for AA treatment in Japanese patients. The present study aimed to assess the association of the baseline NLR with the overall survival (OS) in CPRC patients treated by AA. The present study retrospectively reviewed a total of 90 consecutive patients with CRPC treated with AA from 2011 to 2016 at Yokohama City University Medical Center and National Cancer Center Hospital East. The primary endpoint of the study was the OS, which was defined as the survival from the start of AA administration. The secondary endpoint was the prostate-specific antigen (PSA) response. PSA response was defined as a reduction in antigen levels of $>50 \%$. Complete blood cell counts were performed, and the NLR was calculated using the neutrophil and lymphocyte counts obtained on the same day or a few days prior to the initiation of AA therapy. The NLR cut-off point was determined to be 3.76 for the OS, and divided into the high NLR group of 34 patients and the low NLR group of 56 patients. A PSA response was obtained in 8 patients $(23.5 \%)$ in the high NLR group and in 24 (42.9\%) in the low NLR group. The difference of PSA response between the two groups was significant $(\mathrm{P}=0.037)$. Kaplan-Meier curves demonstrated that a high NLR [NLR $\geq 3.76$; median OS: 8.4 months; $95 \%$ confidence
\end{abstract}

Correspondence to: Dr Takashi Kawahara, Department of Urology and Renal Transplantation, Yokohama City University Medical Center, 4-57 Urafune-cho, Minami-ku, Yokohama 232-0024, Japan

E-mail: takashi_tk2001@yahoo.co.jp

Key words: abiraterone acetate, CRPC, NLR interval (CI): 6.325-10.475 months] was correlated with a risk of mortality compared with a low NLR (NLR <3.76; median OS not reached). A multivariate analysis demonstrated that the NLR was an independent predictor for the OS (hazard ratio: 2.682; 95\% CI: 1.143-6.293; $\mathrm{P}=0.023$ ). The findings suggest that the NLR may be a useful novel biomarker for predicting the prognosis of CRPC patients treated with AA.

\section{Introduction}

Prostate cancer is one of the most frequently diagnosed cancers and a main cause of cancer death in men (1). Screening of prostate-specific antigen (PSA) has contributed to the detection of prostate cancer at an early stage, but prostate cancer is still frequently diagnosed for the first time already at an advanced stage.

In the past decade, several new therapies have been shown to provide a survival benefit in patients with prostate cancer, including abiraterone acetate (AA), enzalutamide, cabazitaxel, radium-223, and sipuleucel $T$ (2). These agents have now become the mainstream treatment for castration-resistant prostate cancer (CRPC). As the choice of treatment has expanded, the establishment of strategies regarding sequence approaches and when to switch agents is crucial for providing the best benefit to CRPC patients.

At present, there are no established biomarkers for predicting the effectiveness of the agents, so doctors must decide on the best treatment approach based on clinical factors. The neutrophil-to-lymphocyte ratio (NLR) is one factor that has been widely reported to be a prognostic factor not only in prostate cancer but also in some solid cancers (3-14). In addition, the NLR can be calculated easily from routine complete blood counts $(\mathrm{CBCs})$ in peripheral blood samples $(4,5)$.

AA, a CYP-17 inhibitor, is now a commonly used in the treatment of CRPC patients. A new biomarker to indicate the best timing for the induction and discontinuation of AA would be useful for clinical practice; however, at the time of writing this report, no such markers have been reported. Thus, previous studies have reported several existing clinical factors as prognostic biomarkers for AA therapy (15-17). The NLR for CRPC treatments has also been investigated in a few reports (18-20), 
but it has not been shown to be a prognostic factor for AA treatment in Japanese patients. We therefore evaluated the association of the NLR with the clinical outcomes, such as PSA response, and overall survival (OS) in CPRC patients receiving AA treatment.

\section{Patients and methods}

Patients. We retrospectively reviewed 90 consecutive patients with CRPC patients treated with AA from 2011 to 2016, at Yokohama City University Medical Center (Yokohama, Japan), Yokohama City University Graduate School of Medicine (Yokohama, Japan), and National Cancer Center Hospital East (Kashiwa, Japan). All patients were administered 1,000 mg of AA orally once a day and $10 \mathrm{mg}$ oral prednisone daily. Nine patients $(10 \%)$ had their dose reduced to $500-750 \mathrm{mg}$ of AA because of adverse events. Patients who had active infectious disease or were treated with steroid for any reason and disease before AA initiation were excluded due to influence to NLR. This study was carried out in accordance with the ethical standards of the Declaration of Helsinki. The study was approved by the review boards of each institution: Yokohama City University Medical Center Institutional Review Board (Yokohama, Japan) and National Cancer Center Hospital East Institutional Review Board (Kashiwa, Japan). Due to the fact that the present study was retrospective and observational, the review board waived the requirement for patient informed consent.

Clinical assessments. The primary endpoint of this study was the OS, which was defined as the survival from the start of AA administration. The secondary endpoint prostate-specific antigen (PSA) response. PSA response was defined as a reduction of more than $50 \%$. AA was administered with $1,000 \mathrm{mg} /$ day plus prednisolone $10 \mathrm{mg}$ /day along with LH-RH agonists. After AA administration, PSA was measured every month, and AA treatment was continued until progression of the disease, as defined by the PCWG2 criteria (21), or unacceptable toxicity. CBCs were performed, and baseline NLR was calculated using the neutrophil and lymphocyte counts obtained on the same day or a few days before the initiation of AA. All data were retrospectively collected from clinical records.

Statistical analyses. We used the area under the receiving operator characteristic (AUROC) curve to determine the cut-off NLR. Median values were used as the cut-off points for other factors (for continuous measurements). Multivariate logistic regression models were used to detect the individual factors. A Kaplan-Meier product limit estimator was used to estimate the OS and PFS. The survival duration was defined as the time between starting AA medication and death of any cause. The log-rank test was performed for comparison. P-values of $<0.05$ were considered to indicate statistical significance. The statistical analyses were performed using the SPSS (IBM, Chicago, IL, USA) and Graph Pad Prism (Graph Pad Software, La Jolla, CA, USA) software programs.

\section{Results}

Patients' characteristics. Table I shows the characteristics of 90 patients. The median initial PSA level was $171.3 \mathrm{ng} / \mathrm{ml}$

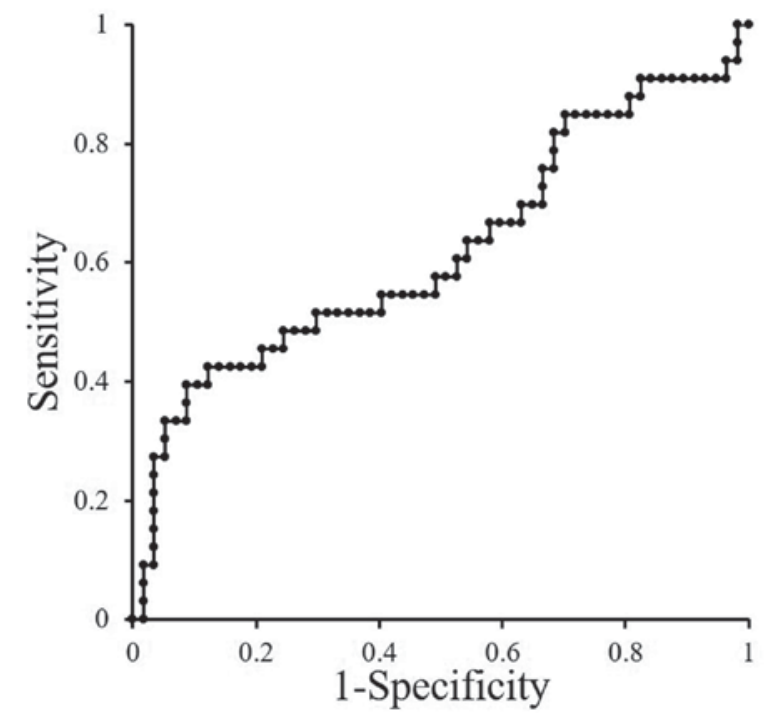

Figure 1. ROC to detect cut-off point of NLR.

(range: 6.1-8770). The Gleason scores were 6 in 1 patient $(1.1 \%), 7$ in 13 patients $(14.4 \%), 8$ in 17 patients (18.9\%), 9 in 39 patients (43.3\%), 10 in 10 patients (11.1\%), and unknown in 10 patients $(11.1 \%)$. For local treatment, 9 patients had prostatectomy (10.0\%), and 8 had radiation therapy (8.9\%). The clinical stages were M1 disease in 61 patients (71.1\%). At the time of AA initiation, the median (mean $\pm \mathrm{SD}$ ) baseline age was $73.2(72.7 \pm 0.9)$, PSA was $55.0 \mathrm{ng} / \mathrm{ml}(307.3 \pm 94.5)$, lactate dehydrogenase (LDH) was $210 \mathrm{IU} / 1(376.0 \pm 68.9)$, and alkaline phosphatase (ALP) was 314 IU/l (557.0 \pm 79.6$)$.

NLR cut-off and correlation with the PSA response and OS. Based on the AUROC curve, the cut-off NLR for OS was determined to be 3.76 , as an NLR of 3.76 showed the highest sensitivity and specificity (Fig. 1). There were 34 patients in the high NLR $(\geq 3.76)$ group and 56 in the low NLR $(<3.76)$ group. A PSA response was obtained in 8 patients (23.\%) in the high NLR group and 24 (42.9\%) in the low NLR group. There was a statistically significant difference between the two groups in the PSA response to AA ( $\mathrm{P}=0.037)$.

We also compared the OS in patients with high vs. low NLRs. Kaplan-Meier curves showed that a high NLR (NLR 23.76; median OS: 8.4 months, $95 \%$ confidence interval [CI]: 6.325-10.475 months) was correlated with a risk of mortality compared with a low NLR (NLR <3.76; median OS not reached) (Fig. 2, Table II).

Multivariate analyses of the $O S$ at AA induction. The results of a multivariate analysis to identify risk factors for the OS at AA induction are shown in Table II. The baseline LDH ( $>210$ IU/l vs. $\leq 210$, hazard ratio: $2.600,95 \%$ CI: 1.018-6.637, $\mathrm{P}=0.046)$ and baseline NLR ( $>3.76$ vs. $\leq 3.76$, hazard ratio: $2.682,95 \%$ CI: $1.143-6.293, \mathrm{P}=0.023$ ) were significant predictors of the OS.

\section{Discussion}

Remarkable advances in CRPC treatment over the past decade have improved the survival of CRPC patients. However, due 
Table I. Patients' background.

\begin{tabular}{|c|c|c|c|}
\hline \multirow[b]{2}{*}{ Variables } & \multicolumn{3}{|c|}{ Number $(\%)$ or median (mean \pm standard deviation) } \\
\hline & Total & NLR $<3.76$ & NLR $\geq 3.76$ \\
\hline Number & 90 & $56(62 \%)$ & $34(38 \%)$ \\
\hline Age (years) & $73.2(72.7 \pm 0.9)$ & $73.0(72.2 \pm 1.0)$ & $75.5(73.5 \pm 1.7)$ \\
\hline \multicolumn{4}{|l|}{ Gleason score } \\
\hline$\leq 6$ & 1 & 1 & 0 \\
\hline 7 & 13 & 7 & 6 \\
\hline$\geq 8$ & 66 & 42 & 24 \\
\hline Unknown & 10 & 6 & 4 \\
\hline Prostatectomy & 9 & 7 & 2 \\
\hline Curative radiation & 11 & 8 & 3 \\
\hline Distant metastasis & 61 & 39 & 22 \\
\hline PSA (ng/ml) & $55.0(307.3 \pm 94.5)$ & $32.57(339.1 \pm 147.0)$ & $80.7(255.8 \pm 70.5)$ \\
\hline LDH (IU/1) & $210(376.0 \pm 68.9)$ & $199.5(327.2 \pm 66.4)$ & $241(456.4 \pm 146.5)$ \\
\hline ALP (IU/1) & $314(557.0 \pm 79.6)$ & $277(527.6 \pm 80.0)$ & $349(604.2 \pm 164.7)$ \\
\hline Initial PSA & $171.3(913.4 \pm 194.3)$ & $185.0(1174.5 \pm 290.2)$ & $126.5(481.1 \pm 167.4)$ \\
\hline
\end{tabular}

NLR, neutrophil to lymphocyte ratio; PSA, prostate-specific antigen; alp, alkaline phosphatase; LDH, lactate dehydrogenase.

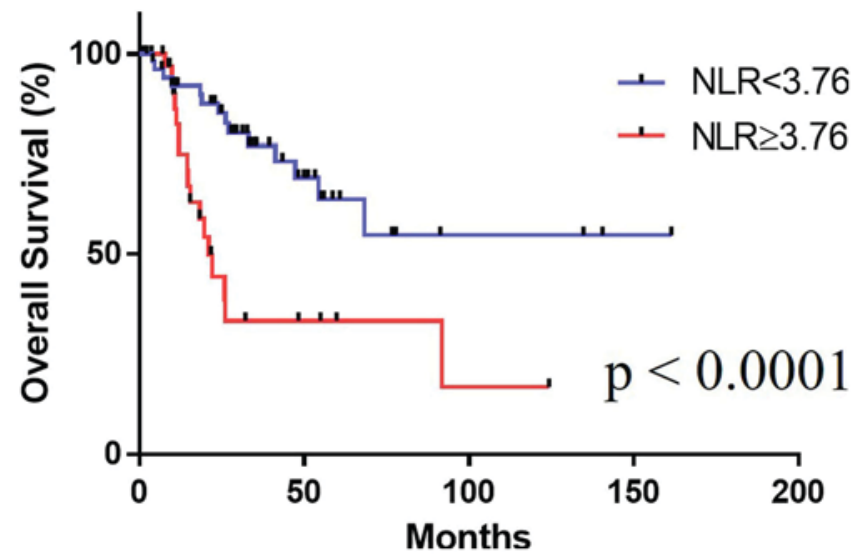

Figure 2. The Kaplan-Meier curve for survival.

to the heterogeneous CRPC population, treatment needs to be individualized further, and determining the optimum sequencing strategy for novel therapies is now the main matter of controversy. The identification of predictive and prognostic factors will aid in deciding whether or not a given treatment is suitable for a patient.

Our investigation showed that a high NLR was a risk factor for not only a poor PSA response but also a poor overall prognosis for AA treatment in CRPC patients. The NLR has been suggested to be an indicator for the general immune response to various diseases, and in cancer, the inflammatory response has been reported to play an important role in the occurrence and development by immune microenvironment in tumor cells $(5,6)$. Previous reports have shown the NLR to be a parameter reflecting the efficacy of treatments and the prognosis in prostate cancer $(9,22)$. We previously reported that the pretreatment NLR in cases of metastatic prostate cancer predicts
Table II. Multivariate analyses.

\begin{tabular}{lcccc}
\hline & HR & $\begin{array}{c}95 \% \mathrm{CI} \\
\text { lower }\end{array}$ & $\begin{array}{c}95 \% \mathrm{CI} \\
\text { upper }\end{array}$ & P-value \\
\hline NLR $\geq 3.76$ & 2.506 & 1.106 & 5.68 & 0.028 \\
ALP $\geq$ median & 1.545 & 0.632 & 3.773 & 0.340 \\
LDH $\geq$ median & 2.607 & 1.021 & 6.655 & 0.045 \\
Initial PSA $\geq$ median & 0.754 & 0.346 & 1.641 & 0.476 \\
Gleason score $(\geq 8)$ & 0.883 & 0.277 & 2.81 & 0.833 \\
PSA $\geq 55.0$ & 1.112 & 0.472 & 2.617 & 0.808 \\
Pre docetaxcel & 0.948 & 0.398 & 2.26 & 0.904 \\
Age $\geq$ median & 1.091 & 0.475 & 2.506 & 0.838 \\
\hline
\end{tabular}

CI, confidence interval; HR, hazard ratio; NLR, neutrophil to lymphocyte ratio; PSA, prostate-specific antigen; alp, alkaline phosphatase; LDH, lactate dehydrogenase.

both the cancer-specific survival and OS (4). Lorente et al also reported that the baseline NLR is a prognostic factor for second-line chemotherapy against metastatic CRPC (9). With regard to AA, several studies have reported a correlation with the NLR in patients with metastatic CRPC. Similar to the result of our study, these studies showed that low baseline NLR was shown to be associated with improved survival; and also low baseline platelet-lymphocyte ratio was similarly associated with better survival $(18,23,24)$. These findings show that the systemic immune system has an impact on the prognosis of CRPC patients, including those treated with AA.

Various mechanisms have been suggested to lead to the epithelial-to-mesenchymal transition, including an increased supply of growth factors, survival factors, pro-angiogenic 
factors, extracellular matrix-modifying enzymes (which can facilitate invasion and metastasis), and inductive signals $(25,26)$. Cho et al reported that patients with an elevated NLR exhibit a relative lymphocyte-mediated immune response to malignancy, thereby worsening their prognosis and increasing the potential for tumor progression $(27,28)$. However, none of the mechanisms reported to underlie the link between the NLR and cancer progression have been widely accepted. The reason why a high baseline NLR leads to a worse prognosis in CRPC patients treated with AA is also unclear; however, the use of prednisone may be associated with this outcome. Boegemann found that baseline a NLR of $>5$ and an NLR-change to $<5$ at eight weeks after the induction of AA was associated with worse survival (23). They considered that the use of prednisone might be associated with this outcome. Prednisone is known to increase the neutrophil count by mobilization from the marginated pool and bone marrow release (29). However, the progression of bone metastasis under AA treatment leads to a dysfunction in the production of neutrophils in the bone marrow, and results in a decreased neutrophil count. They suggested that the administration of prednisone to patients treated with AA might have a strong impact on immunomodulation (23).

This study has several limitations. First, this study was retrospective in nature. As a result, the data for some of the variables were missing. Second, our population was relatively small, heterogeneous, included patients with both metastatic and non-metastatic CRPC and the observation period was relatively short. Our previous report showed that the initial PSA level was higher in the high NLR group (4); however, in this study, the PSA was higher in the low NLR group. The evaluation of a larger patient population over a longer period of time is needed to verify the NLR as a prognostic factor. Third limitation is that as several clinicians treated the patients and the indications for imaging were different, we could not assess the progression using bone scintigraphy or computed tomography. Finally, several treatments for CRPC have been developed, including docetaxel, enzalutamide, AA, Ra-223, and cabazitaxel, and in the near future, other medications, such as ARN-509, ODM-201, and immunosuppressive agents, will become available for CRPC patients. No treatment sequence has been established in CRPC patients. As such, further studies are needed to determine how to use the NLR for clinical benefit.

In conclusion, our findings suggest that the NLR may be a useful new biomarker for predicting the prognosis of CRPC patients treated with AA.

\section{Acknowledgements}

Not applicable.

\section{Funding}

The present study was supported by grants from KAKENHI (grant no. 16K20152) from the Ministry of Education, Culture, Sports, Science and Technology of Japan.

\section{Availability of data and materials}

The analyzed data sets generated during the study are available from the corresponding author on reasonable request.

\section{Authors' contributions}

Conceived and designed the experiments: MY, YH, TK, NM, HU. Analyzed data: MY, TK. Performed the experiments: MY, YH, TK, YK, YM, NM, HU. Wrote the paper: MY, TK, NM, HU.

\section{Ethics approval and consent to participate}

The study was approved by the review boards of each institution: Yokohama City University Medical Center Institutional Review Board (Yokohama, Japan) and National Cancer Center Hospital East Institutional Review Board (Kashiwa, Japan).

\section{Consent for publication}

The present study obtained consent for publication from Yokohama City University Medical Center Institutional Review Board.

\section{Competing interests}

The authors declare that they have no competing interests.

\section{References}

1. Siegel RL, Miller KD and Jemal A: Cancer statistics, 2015. CA Cancer J Clin 65: 5-29, 2015.

2. Heidenreich A, Bastian PJ, Bellmunt J, Bolla M, Joniau S, van der Kwast T, Mason M, Matveev V, Wiegel T, Zattoni F, et al: EAU guidelines on prostate cancer. Part II: Treatment of advanced, relapsing and castration-resistant prostate cancer. Eur Urol 65: 467-479, 2014.

3. Cao J,Zhu X,Zhao X, Li XF and Xu R: Neutrophil-to-lymphocyte ratio predicts psa response and prognosis in prostate cancer: A systematic review and meta-analysis. PLoS One 11: e0158770, 2016.

4. Kawahara T, Yokomizo Y, Ito Y, Ito H, Ishiguro H, Teranishi J, Makiyama K, Miyoshi Y, Miyamoto H, Yao M and Uemura H: Pretreatment neutrophil-to-lymphocyte ratio predicts the prognosis in patients with metastatic prostate cancer. BMC Cancer 16: 111, 2016.

5. Gu X, Gao X, Li X, Qi X, Ma M, Qin S, Yu H, Sun S, Zhou D and Wang W: Prognostic significance of neutrophil-to-lymphocyte ratio in prostate cancer: Evidence from 16,266 patients. Sci Rep 6: 22089, 2016.

6. Yin X, Xiao Y, Li F, Qi S, Yin Z and Gao J: Prognostic role of neutrophil-to-lymphocyte ratio in prostate cancer: A systematic review and meta-analysis. Medicine (Baltimore) 95: e2544, 2016.

7. Langsenlehner T, Thurner EM, Krenn-Pilko S, Langsenlehner U, Stojakovic T, Gerger A and Pichler M: Validation of the neutrophil-to-lymphocyte ratio as a prognostic factor in a cohort of European prostate cancer patients. World J Urol 33: 1661-1667, 2015.

8. Conteduca V, Crabb SJ, Jones RJ, Caffo O, Elliott T, Scarpi E, Fabbri P, Derosa L, Massari F, Numico G, et al: Persistent neutrophil to lymphocyte ratio $>3$ during treatment with enzalutamide and clinical outcome in patients with castration-resistant prostate cancer. PLoS One 11: e 0158952, 2016.

9. Lorente D, Mateo J, Templeton AJ, Zafeiriou Z, Bianchini D, Ferraldeschi R, Bahl A, Shen L, Su Z, Sartor O and de Bono JS: Baseline neutrophil-lymphocyte ratio (NLR) is associated with survival and response to treatment with second-line chemotherapy for advanced prostate cancer independent of baseline steroid use. Ann Oncol 26: 750-755, 2015.

10. Unlu O, Petekkaya E, Ozcan B and Altundag K: The association between neutrophil to lymphocyte ratio and survival in metastatic breast cancer patients. J BUON 21: 1322-1323, 2016.

11. Mahsuni Sevinc M, Riza Gunduz U, Kinaci E, Armagan Aydin A, Bayrak S, Umar Gursu R and Gunduz S: Preoperative neutrophil-to-lymphocyte ratio and plateletto-lymphocyte ratio as new prognostic factors for patients with colorectal cancer. J BUON 21: 1153-1157, 2016. 
12. Xiao Y, Xie Z, Shao Z, Chen W, Xie H, Qin G and Zhao N: Neutrophil and lymphocyte counts at diagnosis are associated with overall survival of pancreatic cancer: A retrospective cohort study. Medicine (Baltimore) 95: e5024, 2016.

13. Yao A, Sejima T, Iwamoto H, Masago T, Morizane S, Honda M and Takenaka A: High neutrophil-to-lymphocyte ratio predicts poor clinical outcome in patients with castration-resistant prostate cancer treated with docetaxel chemotherapy. Int J Urol 22: 827-833, 2015.

14. Taguchi S, Nakagawa T, Matsumoto A, Nagase $\mathrm{Y}$, Kawai T, Tanaka Y, Yoshida K, Yamamoto S, Enomoto Y, Nose Y, et al: Pretreatment neutrophil-to-lymphocyte ratio as an independent predictor of survival in patients with metastatic urothelial carcinoma: A multi-institutional study. Int J Urol 22: 638-643, 2015.

15. Chi KN, Kheoh T, Ryan CJ, Molina A, Bellmunt J, Vogelzang NJ, Rathkopf DE, Fizazi K, Kantoff PW, Li J, et al: A prognostic index model for predicting overall survival in patients with metastatic castration-resistant prostate cancer treated with abiraterone acetate after docetaxel. Ann Oncol 27: 454-460, 2016.

16. Nadal R, Tsai HL, Sinibaldi VJ, Paller CJ, Antonarakis ES, Denmeade SR, Carducci MA and Eisenberger MA: Prognostic factors for clinical outcomes in patients with metastatic castration resistant prostate cancer treated with sequential novel androgen receptor-directed therapies. Prostate 76: 512-520, 2016.

17. Facchini G, Caffo O, Ortega C, D'Aniello C, Di Napoli M, Cecere SC, Della Pepa C, Crispo A, Maines F, Ruatta F, et al: Very early PSA response to abiraterone in mCRPC patients: A novel prognostic factor predicting overall survival. Front Pharmacol 7: 123, 2016

18. Lolli C, Caffo O, Scarpi E, Aieta M, Conteduca V, Maines F, Bianchi E, Massari F, Veccia A, Chiuri VE, et al: Systemic immune-inflammation index predicts the clinical outcome in patients with mCRPC treated with abiraterone. Front Pharmacol 7: 376, 2016

19. Sundar S and O'Cathail M: Neutrophil-lymphocyte ratio is prognostic but not predictive of response to Abiraterone in metastatic castration-resistant prostate cancer. JRSM Open 6 : 2054270415611332,2015

20. Leibowitz-Amit R, Templeton AJ, Omlin A, Pezaro C, Atenafu EG, Keizman D, Vera-Badillo F, Seah JA, Attard G, Knox JJ, et al: Clinical variables associated with PSA response to abiraterone acetate in patients with metastatic castration-resistant prostate cancer. Ann Oncol 25: 657-662, 2014

21. Scher HI, Halabi S, Tannock I, Morris M, Sternberg CN Carducci MA, Eisenberger MA, Higano C, Bubley GJ, Dreicer R et al: Design and end points of clinical trials for patients with progressive prostate cancer and castrate levels of testosterone: Recommendations of the prostate cancer clinical trials working group. J Clin Oncol 26: 1148-1159, 2008.
22. Kawahara T, Fukui S, Sakamaki K, Ito Y, Ito H, Kobayashi N, Izumi K, Yokomizo Y, Miyoshi Y, Makiyama K, et al: Neutrophil-to-lymphocyte ratio predicts prostatic carcinoma in men undergoing needle biopsy. Oncotarget 6: 32169-32176, 2015.

23. Boegemann M, Schlack K, Thomes S, Steinestel J, Rahbar K, Semjonow A, Schrader AJ, Aringer M and Krabbe LM: The role of the neutrophil to lymphocyte ratio for survival outcomes in patients with metastatic castration-resistant prostate cancer treated with abiraterone. Int J Mol Sci 18: pii: E380, 2017.

24. Lozano Martinez AJ, Moreno Cano R, Escobar Páramo S, Salguero Aguilar R, Gonzalez Billalabeitia E, García Fernández R, De La Fuente Muñoz I, Romero Borque A, Porras Martinez M, Lopez Soler F, et al: Platelet-lymphocyte and neutrophil-lymphocyte ratios are prognostic but not predictive of response to abiraterone acetate in metastatic castration-resistant prostate cancer. Clin Transl Oncol 19: 1531-1536, 2017.

25. Hanahan D and Weinberg RA: Hallmarks of cancer: The next generation. Cell 144: 646-674, 2011.

26. Hermanns T, Bhindi B, Wei Y, Yu J, Noon AP, Richard PO, Bhatt JR, Almatar A, Jewett MA, Fleshner NE, et al: Pre-treatment neutrophil-to-lymphocyte ratio as predictor of adverse outcomes in patients undergoing radical cystectomy for urothelial carcinoma of the bladder. Br J Cancer 111: 444-451, 2014.

27. Gondo T, Nakashima J, Ohno Y, Choichiro O, Horiguchi Y, Namiki K, Yoshioka K, Ohori M, Hatano T and Tachibana M: Prognostic value of neutrophil-to-lymphocyte ratio and establishment of novel preoperative risk stratification model in bladder cancer patients treated with radical cystectomy. Urology 79: 1085-1091, 2012

28. Cho H, Hur HW, Kim SW, Kim SH, Kim JH, Kim YT and Lee K: Pre-treatment neutrophil to lymphocyte ratio is elevated in epithelial ovarian cancer and predicts survival after treatment. Cancer Immunol Immunother 58: 15-23, 2009.

29. Nakagawa M, Terashima T, D'Yachkova Y, Bondy GP, Hogg JC and van Eeden SF: Glucocorticoid-induced granulocytosis: Contribution of marrow release and demargination of intravascular granulocytes. Circulation 98: 2307-2313, 1998. 